\title{
AN EXPERIMENTAL METHOD FOR THE TRANSIENT INFRARED INSPECTIONS IN SITU OF HISTORICAL BUILDINGS
}

\author{
Cervino T. ${ }^{1}$, F.Fantozzi $^{1}$, S.Filippeschi ${ }^{1}$, Santini T. ${ }^{3}$ \\ ${ }^{1}$ Via G. Pasquali, $14-50135$-Firenze \\ Tel.335.7494163 \\ 2 Dipartimento di Energetica "Lorenzo Poggi" - Università di Pisa \\ Via Diotisalvi 2, 56126 - Pisa Tel. +39.050.2217100 - Fax +39.050.2217150 \\ f.fantozzi@ing.unipi.it, s.filippeschi@ing.unipi.it \\ ${ }^{3}$ STT Studio ThermoTech. S.a.s \\ Via Bottaccio, 28 - 54100 - Massa \\ Tel. 0585.093084 - Fax 0585.091220 \\ t.santini@studiothermotech.it
}

\section{ABSTRACT}

A big number of important informations can be obtained by the knowledge of the wall texture which is hidden under the plaster layer in the historical buildings. A wall of a historical building is usually made of blocks of different shapes, materials and ages and their infrared visualization can really help the historical, architectural and energy analysis. Infrared thermography is widely employed in the historical buildings for outdoor inspections, but the analysis of the internal wall is rare. The goal of a good infrared image of an internal wall is often random or connect to a few good practical rules. No line guides or standard allows to drive the infrared inspection.

The driving idea of this research project is that of using the step heating thermography method in the historical walls analysis by observing the transient wall temperature with a IR Image. This method can obtain quantitative information on the materials hidden under the plaster layers. By observing the infrared image during the transient heating of the wall, stones, brick or different materials "appears" on the image a different times, according how much their thermal diffusivity is different from that of a reference mortar (a mix of "pozzolana" lime and sand $\alpha=5,494 \cdot 10^{-7} \mathrm{~m}^{2} \mathrm{~s}^{-1}$ ).

In previous works the authors have classified by the gap $\Delta \alpha$ between their thermal diffusivity and that of the reference mortar normalized with the reference diffusivity, several building materials which had been used to make the most of historical walls in Tuscany. The "appearing time" increases as the gap $\Delta \alpha$ decreases. Granite or concrete hollow blocks have values of $\Delta \alpha$ of $164 \%$ and $1163 \%$, respectively while a full brick has a gap $\Delta \alpha$ of $2,5 \%$. Inside this range there are all the building materials. The "appearing time" has been defined as the time necessary to visualize a Limit Thermal Contrast (LTC) between the materials which must be detected and a Sound Area. This LTC depends on the different parameters: thermal resolution of the infrared devices, optical characteristics of the lens and the heating mode.

In order to predict the appearing times of the different material and, therefore, to discern them in a IR image, two ways have been covered: a numerical simulation of the wall transient heating and an experimental analysis of the LTC for difference heating methods (convective, irradiative and a mix of them).

The numerical analysis has been made by a explicit finite differences method and the main results has been compared with those obtained with a finite elements commercial code. 
Figure 1 shows the evolution over time of the LTC for different couples of materials as the plaster thickness increases. The LTC has been fixed to $1.5 \mathrm{~K}$ and a irradiative heating is considered. It is possible to note that for a plaster thickness of $30 \mathrm{~mm}$, the tuff stone has an appearing time of $3000 \mathrm{~s}$, the granite of $3300 \mathrm{~s}$, the basalt stone of $5200 \mathrm{~s}$ and lastly the concrete of $7500 \mathrm{~s}$. This means which in a thermographic inspection will be hard to detect if the granite or the tuff stone, while will be easier to detect a concrete or a basalt stone.

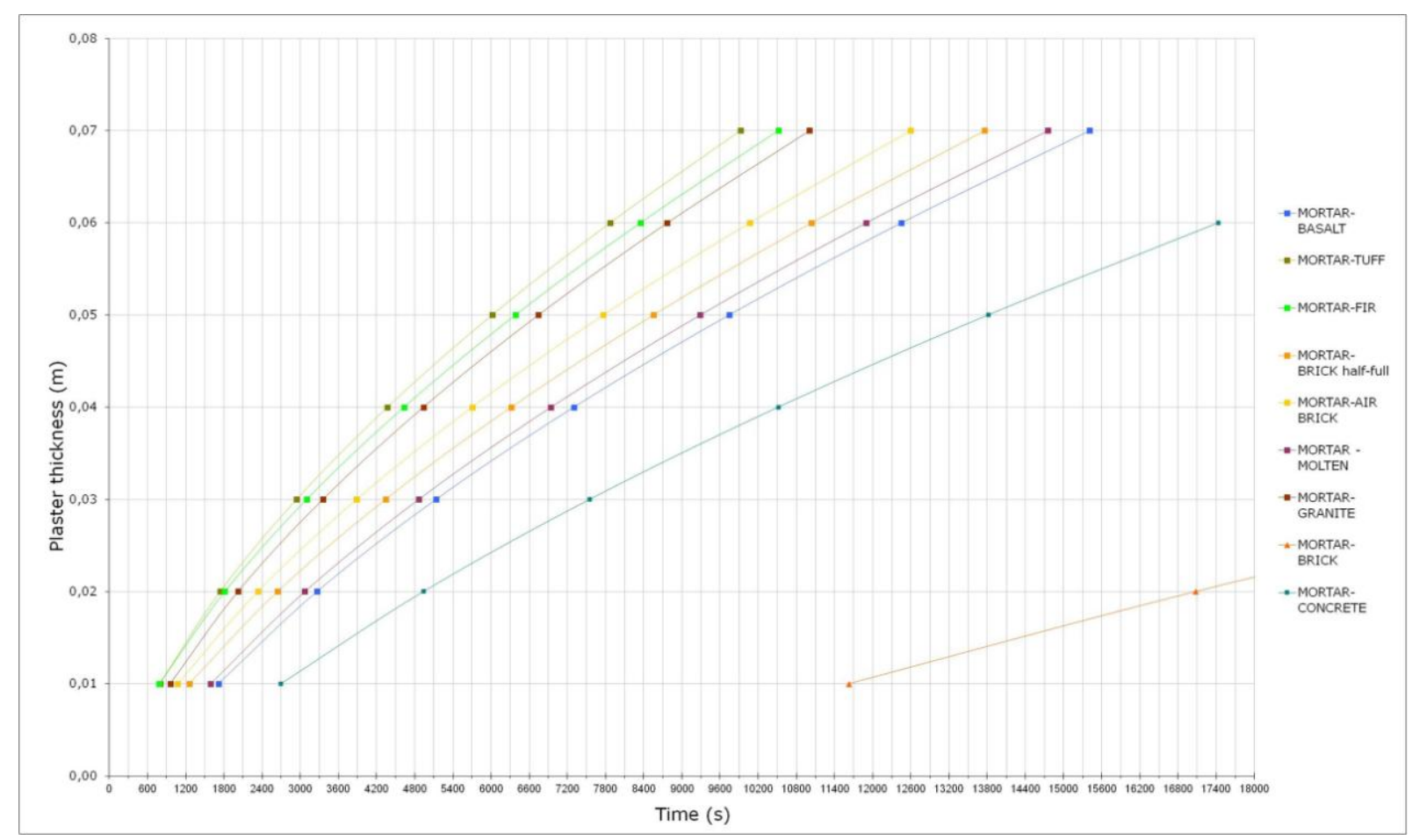

Figure.1- Thermal contrast limit for different couplet of materials and different plaster thickness.

In order to obtain quantitative results, a massive experimental analysis must be carried out, by taking into account different heating modes which deeply affect the results and the LTD.

Two different heater had been used on a reference wall of an historical building made of stone of unknown thermal properties a convective heater and an infrared lamp. The running contrast has been detected with a microbolometric FPA infrared thermocamera (320x240, IFOV 1,4 mm/m, thermal resolution $0.15 \mathrm{~K}$ ) both during the heating time and during the thermal realising time. The thermal contrast which gives a sharp IR image has been experimentally estimated in $0.63 \mathrm{~K}$. The running contrast has been measured at different distance of the heater from the wall. A quantitative characterisation of the heating mode of a reference wall has been made. As example figure 2 shows the running thermal contrast during the heating and the thermal realising time obtained for 4 different stones in the case of a convective heating 


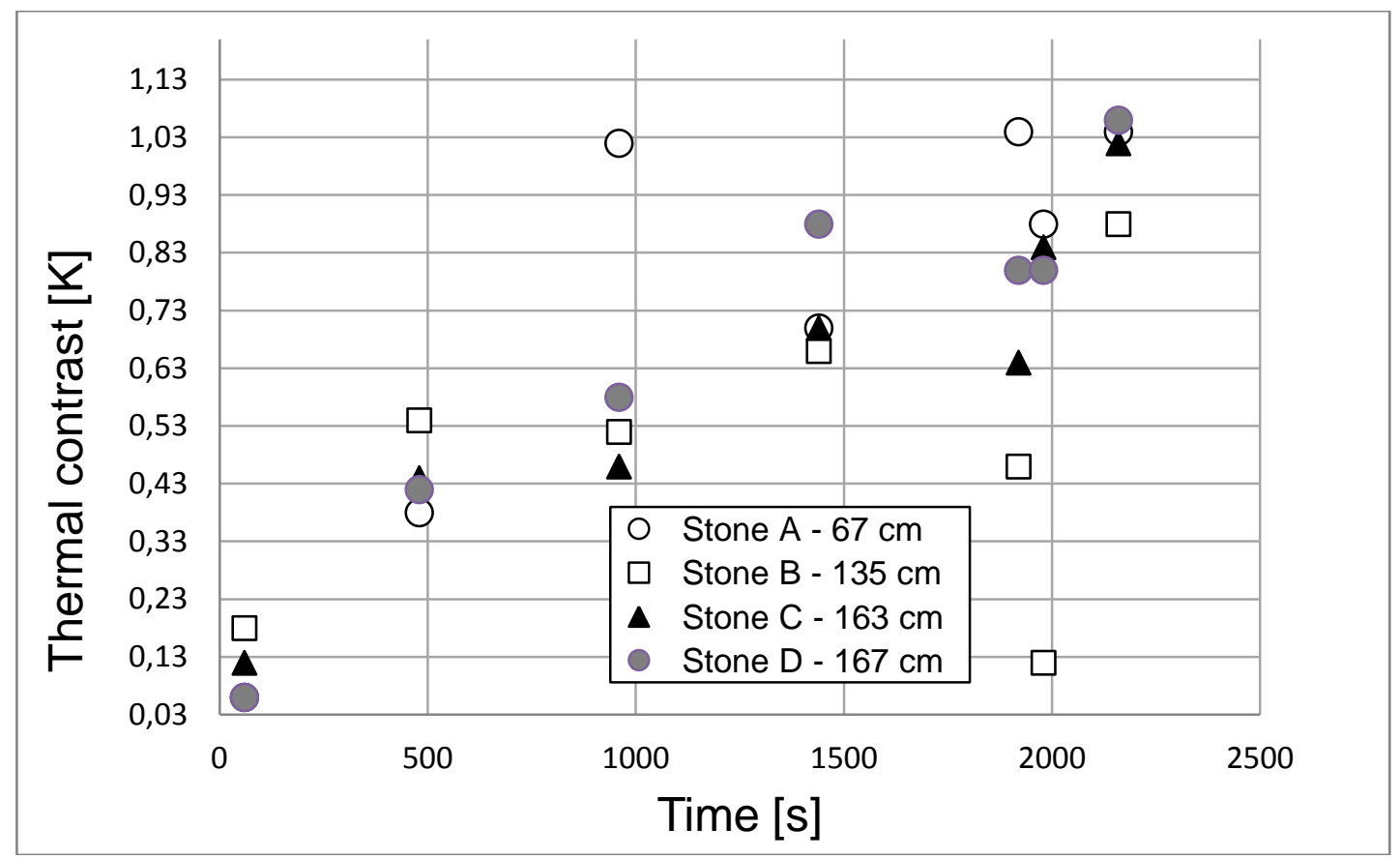

Figure 2- Running Thermal Contrast for 4 different stones during a convective heating 